\title{
Relationship of some ultra trace elements with atherosclerosis
}

\section{Abstract}

Background and objective: Trace elements are now measured as possibly having an important role in the pathogenesis of atherosclerosis. This study aimed to investigate the serum rubidium, rhenium, cesium, and boron levels in patients with atherosclerosis as compared with the control group as well as to detect the effect of age and gender and estimate the correlation among the parameters.

Methods: This case-control study included 40 patients and 40 apparently healthy adults matched age and gender as a control group. Serum parameter levels were estimated using atomic absorption spectrophotometer.

Results: The data of the current study indicated that the levels of rubidium and boron were significantly reduced and there was a significant elevation in the serum level of rhenium while there was no statistical difference in the level of cesium in patients as compared with the control group. In addition, the effect of age and gender did not reveal any significant effect on the serum studied ultra-trace elements levels. There was a negative significant weak correlation between age and $\mathrm{Rb}(r=-0.38, P=0.016)$.

Conclusion: Boron and rubidium were significantly reduced; therefore, supplementation could be important for therapy of atherosclerosis.

Keywords: Atherosclerosis; Boron; Cesium; Rhenium; Rubidium.

\section{Introduction}

Atherosclerosis is a disease in which plaque builds up inside the blood vessels which carry the oxygen to the heart and other parts of the body. Hardening of the arteries occurs when the normal lining cells of the arteries deteriorate, that is mean the wall of the arteries become thick due to deposition of the fatty materials, calcium which lead to building up the plaque narrowing or blockage of the arteries. Atherosclerosis is also known as an arteriosclerotic vascular disease (ASVD) in which the artery wall thickens as a result on invasion and accumulation of white blood cells (macrophages and foam cells) and proliferation of intimal- smooth muscle cell creating an atheromatous (fibrofatty) plaque. Atherosclerosis is a chronic inflammatory disease and inflammation is an important factor of atherosclerotic plaque rupture. ${ }^{1}$ The possible association between trace element (TE) serum levels and cardiovascular diseases (CVDs) including atherosclerosis was studied on the basis of indications that TEs may be directly or indirectly influence the CVD processes. There is a relation between the normal function cardiovascular system with endothelial and erectile dysfunctions. Therefore, the persistence of good cardiovascular health may induce a good quality of life. ${ }^{2}$ While atherosclerosis affects both genders, heart disease, and stroke have occurred a 10-20 year lag in the first atherosclerotic event in females as compared to males. ${ }^{3}$ The Framingham Heart Study of Atherosclerosis investigated that early menopause increases the chance of atherosclerosis. ${ }^{4}$ Trace elements have an important role in atherosclerosis of peripheral arterial occlusive disease ${ }^{.5}$ Also, the trace minerals have a role in persisting a normal structure of cells,

* Department of Clinical Analysis, College of Pharmacy, Hawler Medical University, Erbil, Iraq. 
normal physiology and playing important roles in a status of CVDs. ${ }^{6}$ Normal values help in the recognition the health state of the population, therapy of disease, ${ }^{7}$ and they are important to evaluate the occupational and environmental exposures. $^{8}$ Trace elements (TE) are necessary to the body of the human being, they are related to the physiology of the body ${ }^{9}$ as well as they are considered as an essential part of enzymes, hormones and vitamins. ${ }^{10}$ Deficiencies or extra amounts of TEs become harmful to the health of the body. ${ }^{11}$ Boron (B) is used as a preventative and chemotherapeutic agent and prevent cancer disease by decreasing cell viability and improve cancer cell apoptosis and suppress tumor growth. ${ }^{12}$ Boron, is an element that decreases the cellular damage caused by oxidative stress (OS). ${ }^{13}$ Free radicles (FRs) initiate cell reactive oxygen species (ROS) induced cell damage and cell death. ${ }^{14}$ Cerium oxide $(\mathrm{CeO} 2)$ also has an antibacterial activity ${ }^{15}$ due to the redox potential of $\mathrm{CeO} 2$, the oxidation state of $\mathrm{Ce}$ changed from $\mathrm{Ce}^{3+}$ and $\mathrm{Ce}^{4+}$ depending on the external environment, this redox potential facilitates the inhibiting the bacterial growth and scavenging FRs. $^{16}$ It was published the effect of cesium in the treatment of various kinds of cancers. ${ }^{17}$ Cesium oxide has antioxidant activity, depending on its antioxidant activity, it has emphasized that $\mathrm{CeO} 2$ has the potential effect of treating various types of diseases. $\mathrm{CeO} 2$ initiates cytotoxicity in cancer cells and apoptosis. ${ }^{18}$ $\mathrm{CeO} 2$ nanoparticles decreased DNA damage and facilitating repair. ${ }^{19}$ Transient genotoxicity was also observed from exposure to $\mathrm{CeO} 2$ nanoparticles. ${ }^{20}$ Rhenium $(\mathrm{Re}), 188 \mathrm{Re}$ with pamidronate (3-amino hydroxyl propylidene-1,1bisphosphonic acid) (PMA), because of its biological capabilities it can be used for the treatment of bone pain. ${ }^{21}$ Rhenium is a water-soluble element reacting one atom of $\mathrm{Re}$ and two atoms of selenium has been revealed a strong activity against several kinds of cell lines of a solid tumor. In vitro, of toxicity. ${ }^{22}$ The heavy metal $\operatorname{Re}$ has very low toxicity in human on the basis of the oxidation status (1-7), and this might authorize oxidative detoxification processes. $^{22}$ It was published that Re-based drugs can be directed toward the malignant cells than the normal cells. ${ }^{23,24}$ Rubidium-82 emission tomography has a role in health care and evaluation of patients with coronary artery disease (CAD). ${ }^{25}$ In 2015, It has been tested the anti-depressant activity of rubidium chloride $(\mathrm{RbCl})$ in male mice. ${ }^{26}$ Complex $[\operatorname{Re}(\mathrm{CO}) 3(\mathrm{DIP})$ (py-3-CH2Cl)]+ containing thiol-reactive chloro methyl pyridyl compound for mitochondria immobilization investigates a cytotoxicity and selectivity against cancer cells which initiates damage of the mitochondria and inhibition of the mitochondrial respiration, exhaustion of cellular ATP, as well as increased the levels of reactive oxygen species (ROS). ${ }^{27}$ This study aimed to investigate the serum rubidium, rhenium, cesium, and boron levels in patients with atherosclerosis as compared with the control group as well as to detect the effect of age and gender and estimate the correlation among the parameters.

\section{Methods}

\section{Study design}

This case-control study was performed at College of Pharmacy, Hawler Medical University from March 2014 to May 2015. It included 40 patients of both genders (15 males and 25 females) with atherosclerosis as well as 40 apparently healthy adults matched age and gender as a control group which was selected from Erbil Teaching Hospital. The control group was confirmed to be normal by biochemical and hematological examinations. Patients with atherosclerosis were diagnosed by the specialist physician (cardiologist) after clinical examination and confirmed by laboratory tests in Erbil Teaching Hospital. The serum levels of $\mathrm{Rb}, \mathrm{Cs}, \mathrm{Re}$, and $\mathrm{B}$ were measured using Atomic Absorption Spectrophotometer at Ministry of Science 
and Technology (Chemistry Research Unit) in Baghdad city. All procedures were in accordance with the established ethical standards. All chemicals were highly purified and at analytical grade. The Ethics Committee of Medical Research at the College of Pharmacy, Hawler Medical University approved the study protocol. Oral consent was obtained from all participants before starting the study.

Exclusion criteria: All the patients were free from any diseases except atherosclerosis, and this was confirmed clinically and by hematological and biochemical investigations. All the participants were non-smokers and non alcohol consuming.

Sample collection: A convenience sampling method was used to collect eight $\mathrm{ml}$ of the fasting blood samples have been drawn and left for 30 minutes for coagulation, centrifuged for 15 minutes at 2500-3500 rpm. The sera of the participants have been separated and put them into several plastic plain tubes for the analysis. The sera of the patients and control groups have been stored at $\left(-80 \mathrm{C}^{\circ}\right)$ at Medical Research Center, Hawler Medical University till the day of the analysis. The Frozen sera have been warmed at room temperature.
Statistical analysis: The statistical study has been done using the statistical package for the social sciences SPSS version (18). A $P$ value of $\leq 0.05$ was considered as statistically significant. The numerical data of two groups (patients and controls) were compared using t-test. Analysis of variance (ANOVA) was used to compare the means of four groups. In addition, the Pearson correlation coefficient was used to measure the degree of association between two variables which was estimated in the sample by $r$ value $(-1$ to +1$)$.

\section{Results}

\section{Subjects characteristics:}

The results presented were based on the analysis of the case-control study design. A sample of 40 cases with atherosclerosis and another sample of 40 age and gender-matched apparently healthy control. Table 1 shows the general characteristics of the studied groups. There was a significant decrease in the levels of $\mathrm{B}$ and $\mathrm{Rb}(\mathrm{B}, \mathrm{Rb} P<0.001)$ and there was a significant elevation in the concentration of $\operatorname{Re}(\operatorname{Re} P<0.001)$. There was no significant variation in the serum level of Cs in the patients group as compared with the control group (Cs $P=0.105)$ (Table 2).

Table 1: The general characteristics of the studied groups.

\begin{tabular}{lcccc}
\hline Parameters & $\begin{array}{c}\text { Controls } \\
\text { No.40 }\end{array}$ & $\begin{array}{c}\text { Patients } \\
\text { No.40 }\end{array}$ & Percent\% & Valid Percent \\
\hline Mean age (years) & 52 & 52.4 & & \\
No. of Women & 25 & 25 & 62.5 & 62.5 \\
No. of men & 15 & 15 & 37.5 & 37.5 \\
& & & 100 & 100 \\
\hline
\end{tabular}

Table 2: The serum Levels of $\mathrm{Rb}, \mathrm{Cs}, \mathrm{Re}$, and $\mathrm{B}$ in patients and control groups.

\begin{tabular}{lcccccc}
\hline $\begin{array}{l}\text { Trace element } \\
\mathbf{p p m}\end{array}$ & \multirow{N}{*}{$\mathbf{N}$} & \multicolumn{2}{c}{ Patients } & \multicolumn{2}{c}{ Controls } & \multirow{2}{*}{ Mean } \\
\hline $\mathrm{Rb}$ & 40 & 23.066 & $\mathbf{\pm S D}$ & Mean & $\mathbf{\pm S D}$ & \multirow{P}{*}{ value } \\
$\mathrm{Cs}$ & 40 & .465 & .096 & 37 & 2.150 & $<0.001$ \\
$\mathrm{Re}$ & 40 & 46.298 & 9.996 & 28 & 1.581 & $<0.001$ \\
$\mathrm{~B}$ & 40 & 22.600 & 5.476 & 43 & 2.866 & $<0.001$ \\
\hline
\end{tabular}


This study aimed to investigate the effect there were no statistical variations between of other confounding factors like age and gender. The effect of the age on the serum levels of $\mathrm{Rb}, \mathrm{Cs}, \mathrm{Re}$, and $\mathrm{B}$ (Table 3 ) and different age groups of patients with atherosclerosis, so there was no age effect.

Table 3: The Serum Levels of $\mathrm{Rb}, \mathrm{Cs}, \mathrm{Re}$ and Bin atherosclerotic patients with different age groups.

\begin{tabular}{|c|c|c|c|c|c|}
\hline & Age groups & $\mathbf{N}$ & Mean & SD & $P$ value \\
\hline \multirow[t]{5}{*}{$\mathrm{Rb}$} & $<40$ & 6 & 25.322 & 4.869 & \multirow{5}{*}{0.265} \\
\hline & $40-49$ & 9 & 23.550 & 3.124 & \\
\hline & $50-59$ & 13 & 21.238 & 4.598 & \\
\hline & $60+$ & 12 & 23.553 & 4.633 & \\
\hline & Total & 40 & 23.066 & 4.430 & \\
\hline \multirow[t]{5}{*}{ Cs } & $<40$ & 6 & .427 & .117 & \multirow{5}{*}{0.575} \\
\hline & $40-49$ & 9 & .460 & .081 & \\
\hline & $50-59$ & 13 & .459 & .069 & \\
\hline & $60+$ & 12 & .493 & .122 & \\
\hline & Total & 40 & .465 & .096 & \\
\hline \multirow[t]{5}{*}{$\operatorname{Re}$} & $<40$ & 6 & 49.788 & 5.480 & \multirow{5}{*}{0.254} \\
\hline & $40-49$ & 9 & 49.408 & 6.119 & \\
\hline & $50-59$ & 13 & 46.735 & 10.991 & \\
\hline & $60+$ & 12 & 41.748 & 12.010 & \\
\hline & Total & 40 & 46.298 & 9.996 & \\
\hline \multirow[t]{5}{*}{ B } & $<40$ & 6 & 23.000 & 6.419 & \multirow{5}{*}{0.784} \\
\hline & $40-49$ & 9 & 22.444 & 4.773 & \\
\hline & $50-59$ & 13 & 21.462 & 5.502 & \\
\hline & $60+$ & 12 & 23.750 & 5.910 & \\
\hline & Total & 40 & 22.600 & 5.476 & \\
\hline
\end{tabular}


Table 4 shows that there were no statistical There was a negative, weak significant variations between atherosclerotic females and males, so there was no gender effect. correlation between $\mathrm{Rb}$ and age $(r=-0.38$, $P=0.016)$ as shown in Table 5.

Table 4: The serum levels of $\mathrm{B}, \mathrm{Cs}, \mathrm{Rb}$, and, Rh in atherosclerotic females and males.

\begin{tabular}{llccccc}
\hline & GENDER & $\mathbf{N}$ & Mean & $\mathbf{\pm S D}$ & SE & $\boldsymbol{P}$ value \\
\hline AGE & Female & 25 & 50.240 & 10.670 & 2.134 & \\
& Male & 15 & 56.000 & 8.203 & 2.118 & 0.081 \\
$\mathrm{Rb}$ & Female & 25 & 23.236 & 4.635 & .927 & \\
& Male & 15 & 22.782 & 4.208 & 1.087 & 0.758 \\
$\mathrm{Cs} \quad$ Female & 25 & .458 & .100 & .020 & \\
& Male & 15 & .477 & .092 & .024 & 0.551 \\
$\mathrm{Re}$ & Female & 25 & 47.733 & 8.213 & 1.643 & \\
& Male & 15 & 43.907 & 12.366 & 3.193 & 0.298 \\
$\mathrm{~B}$ & Female & 25 & 23.760 & 5.840 & 1.168 & \\
& Male & 15 & 20.667 & 4.320 & 1.116 & 0.084 \\
\hline
\end{tabular}

Table 5: Correlation coefficient among age, B, Cs, Rb, and, Rh (N=40).

\begin{tabular}{llccccc}
\hline & AGE & Rb & Cs & Rh & B \\
\hline AGE & Pearson Correlation & 1 & -0.075 & 0.296 & $-.380^{*}$ & 0.071 \\
& Sig. (2-tailed) & & 0.645 & 0.064 & 0.016 & 0.665 \\
Rb & Pearson Correlation & -0.075 & 1 & 0.008 & 0.043 & 0.227 \\
& Sig. (2-tailed) & 0.645 & & 0.963 & 0.794 & 0.159 \\
& Pearson Correlation & 0.296 & 0.008 & 1 & -0.220 & -0.033 \\
& Sig. (2-tailed) & 0.064 & 0.963 & & 0.173 & 0.838 \\
Re & Pearson Correlation & $-0.380^{*}$ & 0.043 & -0.220 & 1 & 0.019 \\
& Sig. (2-tailed) & 0.016 & 0.794 & 0.173 & & 0.909 \\
& Pearson Correlation & 0.071 & 0.227 & -0.033 & 0.019 & 1 \\
& Sig. (2-tailed) & 0.665 & 0.159 & 0.838 & 0.909 & \\
\hline
\end{tabular}




\section{Discussion}

Certain TEs are essential for the life and health of the human. From previous studies, they are found that there TEs are essential for the metabolism or may have a role on growth or survival, there is an alteration in the levels of TEs during malfunction or destruction of tissue. ${ }^{28}$ Accordingly, these ultra TEs might have a role for detection risk factors, early diagnosis, therapy and prevention of atherosclerosis. From the data of the current study which indicated the Re was positively correlated with atherosclerosis while, $\mathrm{B}, \mathrm{Cs}$, and $\mathrm{Rb}$ were negatively associated with atherosclerosis. The association between TEs concentrations and CVDs indicated that TEs might be directly or indirectly participated in the processes of in CVDs. ${ }^{29}$ It is well known that variations in TEs concentration are potentially associated with adverse clinical outcome.

\section{Boron}

The data of the current study showed that there was a significant reduction in the serum level of $B$ in patients with atherosclerosis as compared with the control group. This result was in harmony with the fact that $B$ has a role in protection against atherosclerosis as a result of the elevation of endogenous estrogen. ${ }^{30}$ Also, $B$ has a role in the regulation of the $B^{30}$ due to its effect on calcium metabolism. ${ }^{31}$ An abnormal calcium metabolism induces plaque formation on the wall of the artery which results from the induction of atherosclerosis. In addition, B has an antioxidant effect which may be contributed to preventing atherosclerosis. ${ }^{32}$ In addition, $B$ containing drugs have a therapeutic effect for atherosclerosis which leads to remove cholesterol from tissues and reduced lipid deposition.

\section{Cesium}

The serum level of Cs was reduced in patients with atherosclerosis as compared with the control group, but the level did not reach the significant value possibly due to the small sample size. The reduction in the serum level of $\mathrm{Ce}$ is because $\mathrm{Ce}$ is considered an antioxidant and it has SOD and catalase mimetic action. ${ }^{18}$

\section{Rhenium}

The serum concentration of $\operatorname{Re}$ was significantly increased in patients group with atherosclerosis as compared with the control group and the explanation for this significant increased might be due the occurrence of atherosclerosis which is considered as an oxidative stress status which promotes the release of $\mathrm{Rh}$ in human circulation.

\section{Rubidium}

The data of the current study has been shown that there was a significant decrease in the serum level of $\mathrm{Rb}$ in patients with atherosclerosis as compared with the control group. The explanation for this significant reduction needs further studies to explore and elucidate this variation in the serum patient levels as compared with the control group. These alterations in the serum levels of the TEs may provide a good opportunity for diagnosis the patients who are at high risk for developing atherosclerosis for correcting these abnormal levels of TEs as well as foxing more light on the benefit of these TEs on the pathophysiology of atherosclerosis. The estimation of these TEs might be used as a complementary tool for diagnosis and therapy of atherosclerosis. This study was the first report dealing with the association of atherosclerosis with these ultra TEs that was not previously published.

\section{Effect of other confounding factors}

In this study, the mean age of the patients at the diagnosis was 52 years and this finding was consistent with Another previous study, which showed that atherosclerosis occurred in men at age 40 years and in women between the ages 50 and 60 years because of the deficiency of estrogen. ${ }^{33}$ These results agreed with the findings of the current study; the average age of atherosclerotic females was (37-71), and the average age of the atherosclerotic males was 
(41-70). Accordingly, it was emphasized that advanced age is considered a risk factor for the occurrence of atherosclerosis. In this study, there was no age effect on the serum levels of measured ultra TEs. There were no significant differences between different age groups concerning the interested ultra TEs. Concerning the confounding of gender, 15 of the atherosclerotic patients were males and 25 of them were females. These findings were not in harmony with a previous study, ${ }^{33}$ which detected that males were considered as a risk factor for atherosclerosis. In the current study, there were also no significant differences between both genders concerning the studied ultra TEs.

\section{Conclusion}

This study is the first to show the potential of the alterations in the serum levels of studied ultra TEs and if repair these variations and returned to the basement serum levels might protect against factor triggered atherosclerosis. Accordingly, these alterations might be indicated that there was a correlation with atherosclerosis. The data of the present study are consistent with the hypothesis that alterations in the serum level of estimated trace elements may contribute and involve to atherosclerosis. Further researches are needed to conclude whether an alteration in the serum levels of focused trace elements might be a risk factor for atherosclerosis.

\section{Competing interests}

The author declares that she has no competing interests.

\section{References}

1. da Silva RM. Influence of Inflammation and Atherosclerosis in Atrial Fibrillation. Curr. Atheroscler Rep 2017; 19(1):2.

2. Lane-Cordova AD, Kershaw K, Liu K, Herrington $D$, Lloyd-Jones DM, Kershaw K, et al. Association between Cardiovascular Health and Endothelial Function with Future Erectile Dysfunction: The Multi-Ethnic Study of Atherosclerosis. Am J Hypertens 2017; 30(8):815-21.
3. Mozaffarian D, Benjamin EJ, Go AS, Arnett DK, Blaha MJ, Cushman M, et al. Heart disease and stroke statistics--2015 update: a report from the American Heart Association. Circulation 2015; 131(4):e29-322.

4. Wellons M, Ouyang P, Schreiner PJ, Herrington DM, Vaidya D. Early menopause predicts future coronary heart disease and stroke: The multi-ethnic study of atherosclerosis. Menopause 2012; 19(10):1081-7.

5. Fazeli B. Trace elements and toxic heavy metals play a role in Buerger's disease and atherosclerotic peripheral arterial occlusive disease. IntAngiol 2011; 30(6):598-9.

6. Islamoglu Y, Evliyaoglu O, Tekbas E, Cil H, Elbey MA, Atilgan Z, et al. The relationship between serum levels of $\mathrm{Zn}$ and $\mathrm{Cu}$ and severity of coronary atherosclerosis. Biol Trace Elem Res 2011; 144(1-3):436-44.

7. Korvela $M$, Lind $A L$, Wetterhall $M$, Gordh $T$, Andersson M, Pettersson J. Quantification of 10 elements in human cerebrospinal fluid from chronic pain patients with and without spinal cord stimulation. J Trace Elements Med Biol 2016; 37:1-7.

8. Bloise A, Barca D, Gualtieri AF, Pollastri S, Belluso E. Trace elements in hazardous mineral fibres. Environ Pollut 2016; 216:314-23.

9. Czarnek K, Terpiłowska S, Siwicki AK Selected aspects of the action of cobalt ions in the human body. Cent Eur J Immunol 2015; 40:236-42.

10. Araya M, Pizarro F, Olivares M, Arredondo M, González M, Méndez M. Understanding copper homeostasis in humans and copper effects on health. Biol Res 2006; 39:183-7.

11. Davis CD. Low dietary copper increases fecal free radical production, fecal water alkaline phosphatase activity and cytotoxicity in healthy men. J Nutr 2003; 133:522-7.

12. Li X, Wang $X$, Zhang J, Hanagata N, Wang $X$, Weng $Q$, et al.Hollow boron nitride nanospheres as boron reservoir for prostate cancer treatment. Nat Commun 2017; 8:13936.

13. Bahadoran $H$, Naghii MR, Mofid M, Asadi MH, Ahmadi K, Sarveazad A. Protective effects of boron and vitamin $\mathrm{E}$ on ethylene glycol-induced renal crystal calcium deposition in rat. Endocr Regul 2016; 50(4):194-206.

14. Pešić M, Podolski-Renić A, Stojković S, Matović $B$, Zmejkoski D, Kojić $V$, et al. Anti-cancer effects of cerium oxide nanoparticles and its intracellular redox activity. ChemBiol Interact 2015; 232:85-93.

15. Babu KS, Anandkumar M, Tsai TY, Kao TH, Inbaraj BS, Chen BH. Cytotoxicity and antibacterial activity of gold-supported cerium oxide nanoparticles. Int J Nanomedicine 2014; 9:5515-31.

16. Babu S, Velez A, Wozniak K, Szydlowska J, Seal S. Electron paramagnetic study on radical scavenging properties of ceria nanoparticles. 
ChemPhys Lett 2007; 442:405-8.

17. Sartori HE. Cesium therapy in cancerpatients. Pharmacol Biochem Behav 1984; 21(Suppl 1):11 -3 .

18. Khan S, Ansari AA, Rolfo C, Coelho A, Abdulla M, Al-Khayal $\mathrm{K}$, et al. Evaluation of in vitro cytotoxicity, biocompatibility, and changes in the expression of apoptosis regulatory proteins induced by cerium oxide nanocrystals. Sci Technol Adv Mater 2017; 18(1):364-73.

19. Caputo F, De Nicola M, Sienkiewicz A, Giovanetti $A$, Bejarano I, Licoccia S, et al. Cerium oxide nanoparticles, combining antioxidant and UV shielding properties, prevent UV-induced cell damage and mutagenesis. Nanoscale 2015; 7(38):15643-56.

20. Franchi LP, Manshian BB, de Souza TA, Soenen SJ, Matsubara EY, Rosolen JM, et al. Cyto- and genotoxic effects of metallic nanoparticles in untransformed human fibroblast. Toxicolln Vitro 2015; 29(7):1319-31.

21. Erfani M, Rahmani N, Doroudi A, Shafiei M. Preparation and evaluation of rhenium-188pamidronate as a palliative treatment in bone metastasis. Nucl Med Biol 2017; 49:1-7.

22. Collery P, Mohsen A, Kermagoret A, Corre S, Bastian G, Tomas A, et al. Antitumor activity of a rhenium (I)-diselenoether complex in experimental models of human breast cancer. Invest New Drugs 2015; 33(4):848-60.

23. Ho J, Lee WY, Koh KJ, Lee PP, Yan YK. Rhenium(I) tricarbonyl complexes of salicylaldehyde semicarbazones: synthesis, crystal structures and cytotoxicity. J Inorg Biochem 2013; 119:10-20.

24. Leonidova A, Gasser G. Underestimated potential of organometallic rhenium complexes as anticancer agents. ACS Chem Biol 2014; 9(10):2180-93.

25. Smith MF. Advances in rubidium PET and integrated imaging with CT angiography. Curr Cardiol Rep 2008; 10(2):135-41.

26. Kordjazy N, Haj-Mirzaian A, Amiri S, Ostadhadi $S$, Kordjazy M, Sharifzadeh $M$, et al. Elevated level of nitric oxide mediates the anti-depressant effect of rubidium chloride in mice. Eur $\mathrm{J}$ Pharmacol 2015; 762:411-8.

27. Yang J, Zhao JX, Cao Q, Hao L, Zhou D, Gan Z, et al. Simultaneously Inducing and Tracking Cancer Cell Metabolism Repression by Mitochondria-Immobilized Rhenium(I) Complex. ACS Appl Mater Interfaces 2017; 9(16):1390012.

28. Henry A. Schroeder. The Role of Trace Elements in Cardiovascular Diseases. Medical Clinics of North America 1974; 58(2):381.

29. Aalbers TG, Houtman JP. Relationships between trace elements and atherosclerosis. Sci Total Environ 1985; 43(3):255-83.

30. Naghii MR, Samman S. The effect of boron supplementation on its urinary excretion and selected cardiovascular risk factors in healthy male subjects. Biol Trace Elem Res 1997; 56(3):273-86.

31. Wilson JH, Ruszler PL. Effects of dietary boron supplementation on laying hens. Br Poul Sci 1996; 37(4):723-9.

32. Lee IP, Sherins R J, Dixon R L. Evidence for induction of germinal aplasia in male rats by environmental exposure to boron. Tox Appl Pharmacol 1978; 45(2):577-90.

33. Richard-Sheppard M, Vinay K, Abbas AK, Nelson F. Robbins Basic Pathology: With student consult online access. $8^{\text {th }}$ ed. Philadelphia: Saunders; 2007. P. 345. 